\title{
Electron-Energy and -Angular Distributions in the Double Photoionization of Helium
}

\author{
R. Wehlitz, F. Heiser, O. Hemmers, B. Langer, A. Menzel, and U. Becker \\ Fritz-Haber-Institut der Max-Planck-Gesellschaft, Faradayweg 4-6, W-1000 Berlin 33, Germany
}

(Received 15 October 1991)

\begin{abstract}
Photoelectron spectra of helium have been measured at different angles and at various photon energies above the double-ionization threshold up to $120 \mathrm{eV}$ to investigate the behavior of the energy and angular distributions of shake-off electrons. Both energy and angular distributions clearly show a U-shaped profile which turns to a flat curve near threshold pointing to a uniform intensity distribution over the kinetic energy for all angles in this excitation energy regime. Our results for the angular-distribution asymmetry parameter indicate qualitative agreement with theoretical predictions but fail to prove them quantitatively.
\end{abstract}

PACS numbers: $32.80 . \mathrm{Fb}$

The role of electron correlation in helium is of fundamental importance in the study of a three-particle system in a Coulomb field. At first, Madden and Codling [1] observed autoionization processes in He using synchrotron radiation; later studies were extended to satellite lines [2]. In both these cases one electron remains near the ion; however, beyond the double-ionization threshold two electrons can leave the atom simultaneously. This results in a continuously distributed energy curve besides the main line and satellite lines, as shown schematically in Fig. 1. In principle, this is also valid for the other rare gases; however, here the spectra are more complicated due to double-ionization thresholds for each coupling of the double-hole state. Furthermore, in the heavier rare gases, satellite and participator Auger lines originating from satellite states are superimposed on the shake-off intensity [3], which makes the interpretation ambiguous. The analysis of electrons emitted in simultaneous double ionization may be complicated even for experiments using coincidence techniques because of the fact that simultaneous and sequential processes do have, in many cases, the same final ionic state. Therefore, in spite of recent progress in coincidence experiments [4], helium is still best suited for a systematic study of the kinetic-energy and angular distributions of shake-off electrons. Note that the previous photoelectron measurements regarding the shake-off phenomenon in $\mathrm{He}, \mathrm{Ne}$, and $\mathrm{Ar}$ [5] were limited to the high-energy part of the shake-off spectrum and gave therefore no direct information on its overall

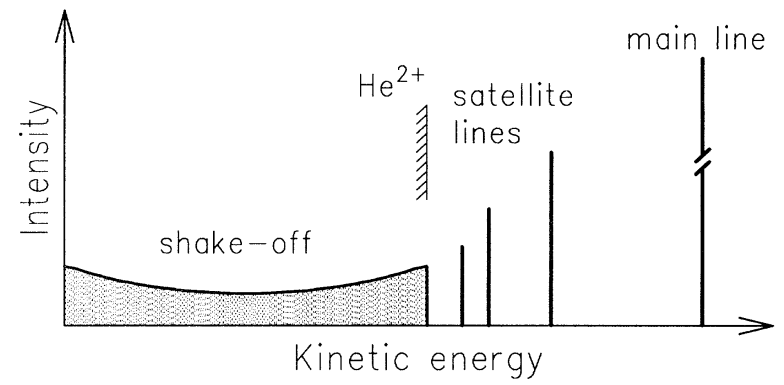

FIG. 1. Schematic photoelectron spectrum of helium above the double-ionization threshold. shape. Theoretically, by using simple screening arguments, the energy distribution between both electrons is expected to be U-shaped; i.e., the faster electron is less attracted to the remaining ion due to the screening of the Coulomb field by the slower electron, which should lead to an enhancement in intensity for the corresponding slow and fast electrons. Calculations by Chang and Poe [6] support this picture and predict a defined dependence of the energy distribution on the photon energy.

Many experiments have been performed concerning the threshold behavior of double photoionization which was first discussed by Wannier [7]. In addition, various theoretical studies apply the Wannier theory to predict, among other things, (i) the energy dependence of the double-ionization cross section, (ii) the energy distribution between the outgoing electrons, and (iii) the angular correlation between these electrons (for references see, e.g., [8,9]). The energy range of validity for Wannier's law is still subject to controversy [8-13]; following more recent publications [8,9] this range is limited to about 2 $\mathrm{eV}$ above the double-ionization threshold.

In this Letter we report on the energy distribution between the two escaping electrons and their angular distribution with respect to the electric vector of the synchrotron light from 2 up to $41 \mathrm{eV}$ above threshold. This noncoincident angular distribution is sensitive to the angular correlation of the two outgoing electrons as shown by Huetz et al. [14]. These authors also show that the angular distribution can be described similar to the case of single photoionization. This implies that there is a "magic" angle where the intensity is independent of the angular distribution, a statement still under dispute [15] because of missing experimental evidence. Therefore a well based understanding of the electron behavior in simultaneous two-electron emission is crucial for all double-ionization studies.

Our experiments were performed at the Hamburger Synchrotronstrahlungslabor (HASYLAB) using photons from a 5.6-m toroidal grating monochromator (TGM) [16] during single-bunch mode operation of the storage ring DORIS II. Our time-of-flight (TOF) spectrometer is mounted on a rotatable chamber with the photon beam on its axis. The spectrometer has a flight path of $679 \mathrm{~mm}$ 
and it is designed for high transmission at low kinetic energies. A small acceleration voltage of $2 \mathrm{~V}$ was applied behind the entrance aperture of the spectrometer enabling, in conjunction with the time window of $960 \mathrm{n}$, the recording of complete electron spectra. More information about the spectrometer system is given elsewhere [17].

Here it is worthwhile to mention that the gas pressure in the drift tube of our spectrometer is reduced compared to the pressure in the interaction region. This is achieved by (i) a collimating gas inlet, (ii) a small entrance aperture of the spectrometer, and (iii) an additional turbomolecular pump for the drift tube. Nevertheless a small nonstatistical background, originating from inelastic scattering, could be seen. In order to determine this background, spectra below the first satellite threshold were recorded and scaled to the $\mathrm{He} 1 s$ main line; an aluminum filter was used to reduce the contribution of higher-order light. Figure 2 shows some of the recorded helium spectra at the magic angle after background subtraction and transmission corrections. It can be seen that the energy distribution of the two outgoing electrons is symmetric to about the center of the shake-off region. At high excess energies the energy distribution is $U$-shaped but turns to a flat distribution when approaching threshold. The measurements of Lablanquie et al. [9] showed that the energy-distribution curve of helium shake-off electrons is flat within $20 \%$ up to at least $15 \mathrm{eV}$ above threshold, which is in contrast to the electron-impact measurements of Pichou et al. [11] whose curve is flat only up to $3.6 \mathrm{eV}$ above threshold. Regarding our spectra the $20 \%$ criterion for flatness is fulfilled between 10 and $21 \mathrm{eV}$ above threshold which is in accordance with the results of Ref. [9]. The curves in the shake-off region are interpolated from calculated curves by Chang and Poe [6] according to their photon energy dependence. The curvature is in good agreement with our data even for the

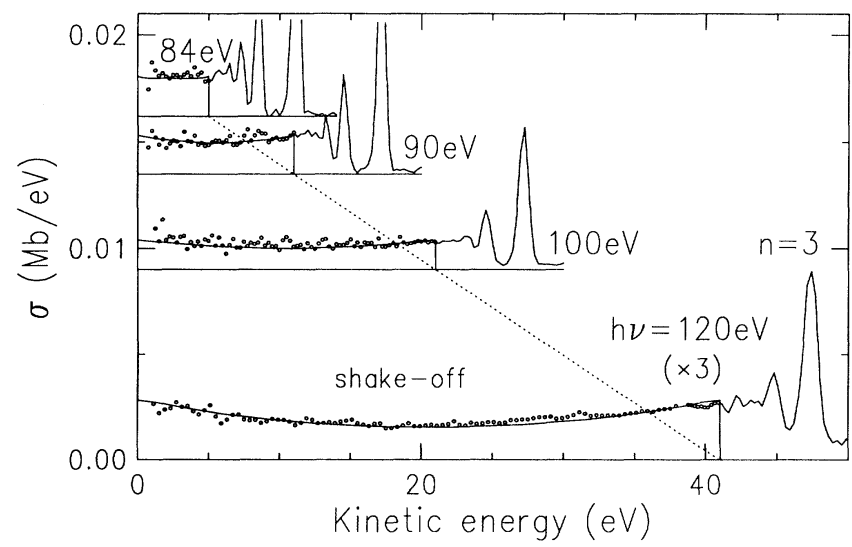

FIG. 2. Photoelectron spectra of helium taken at different photon energies. The solid lines in the shake-off region were interpolated from theoretical curves by Chang and Poe [6]. lowest energy part. This shows that the extended validity range of the flatness criterion $(20 \%)$ is basically due to the general form of the energy distribution of the two outgoing electrons, independent of any specific threshold law. Most other theories also predict a symmetric energy distribution but they are restricted to a certain photon energy [18-20] or a certain range of photon energies [21] so that they cannot be used for comparison with our data. The integral intensity in the shake-off region is twice the value of the double-ionization cross section. Since complete spectra are recorded we also get the single-ionization cross section, so it is possible to calculate the double-to-single photoionization ratio. The result is shown in Fig. 3 together with different ion-yield data [5,8,22-24]. Measurements by Schmidt et al. [22] and Kossmann, Schmidt, and Anderson [8] are in good agreement with our data while the other data show some deviation and are in addition inconsistent with each other.

In order to derive angular distributions, spectra were taken at three different angles which are shown in Fig. 4. The solid lines in the shake-off region are parabolic curves fitted to our data because there is no explicit expression for angle-dependent shake-off curves. At first glance one can see a dependence of the curvature on the angle. This means that the angular-distribution curve of the double-ionization process at a fixed photon energy depends on the kinetic energy of the outgoing electron. The calculation of the angular-distribution parameter $\beta$ [25] by use of the fitted energy-distribution curves gives an angular-distribution curve ( $\beta$ curve) that is $U$-shaped as shown in Fig. 5(a). The same result is obtained when $\beta$ values are derived from energy intervals without special assumptions on the angle-dependent energy-distribution curves. Moreover the $\beta$ curve is dependent on the photon energy with respect to the absolute value and curvature. Near the double-ionization threshold the $\beta$ curves become flat and they converge to an extrapolated value of

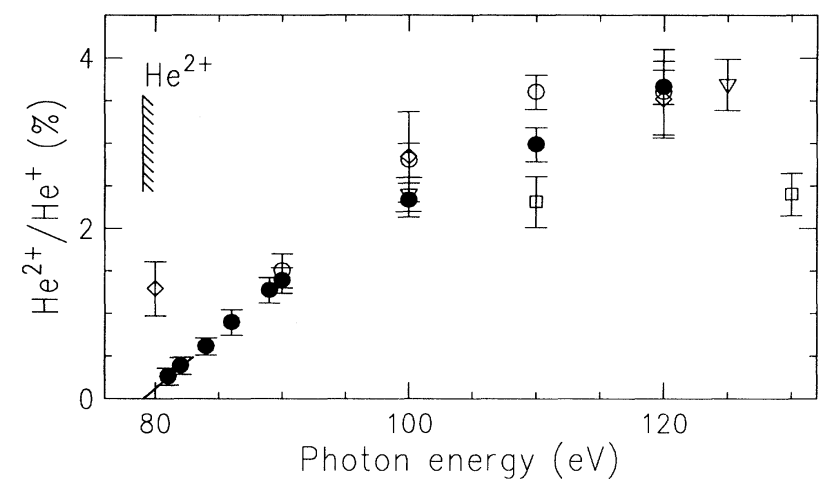

FIG. 3. Double-to-single photoionization ratio of helium determined by photoelectrons (solid circles) in comparison to ion-yield measurements of Carlson [5] (open squares), Schmidt et al. [22] (open triangles), Wight and Van der Wiel [23] (open circles), Holland et al. [24] (open diamonds), and Kossmann et al. [8] (solid line). 


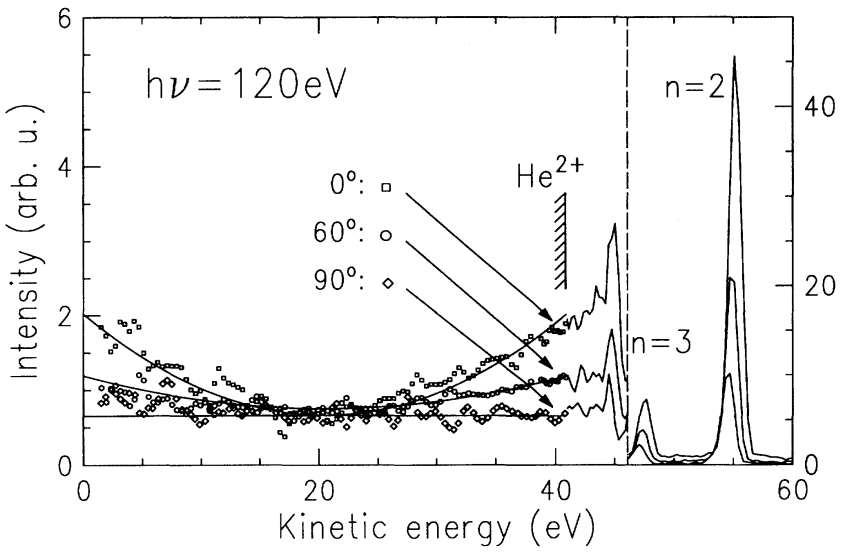

FIG. 4. Helium photoelectron spectra taken at $h v=120 \mathrm{eV}$ at different angles with respect to the plane of the storage ring. The solid lines in the shake-off region are parabolic curves fitted to our spectra disregarding short-range variations which proved to be accidental.

$\beta \approx-0.3$ at threshold. This is in good agreement with recent measurements performed by Hall et al. [13] using a zero-volt spectrometer. Their results are partly displayed in Fig. 5(b) together with calculations by Huetz et al. [14]. The $\beta$ values for different photon energies given by Hall et al. are actually measured at a shake-off kinetic energy of $0.14 \mathrm{eV}$ only. Following these authors it seems to be a reasonable extrapolation, supported by our results concerning the flatness of the $\beta$ curves, to substitute their data points as flat $\beta$ curves for easier comparison with our data. Both sets of experimental data seem to be in contrast to predictions of Green [26] and Wannier [7] who expect $\beta=-1$ at threshold. Theoretical curves by Huetz et al. [14] based on Wannier's law show a possible solution of this problem: The $\beta$ value rises very fast from $\beta=-1$ at threshold to a higher $\beta$ value depending on the angular correlation of the shake-off electrons. Two theoretical curves taking into account different angular correlations between shake-off electrons [14] are depicted in Fig. 5(b). The lower curve is in better agreement than the upper curve which means a stronger angular correlation between shake-off electrons in the near-threshold region. Figure 5 (b) also confirms that the range of validity of Wannier's law does not extend beyond $2 \mathrm{eV}$ above threshold. An explanation for this discrepancy may be the competition between symmetry requirements on the one hand and electron correlation effects on the other hand, as suggested by Hall et al. [13]. The strengths of electron correlations are not only reflected by double ionization but also by satellite lines. Looking at the angular-distribution parameters $\beta$ of the helium satellites with high principal quantum number there is a surprising similarity to the high-kinetic-energy side of the shake-off $\beta$ curves, as shown in Fig. 6 for the satellite $n=4$. This fact supports the assumption made above that the excitation energy
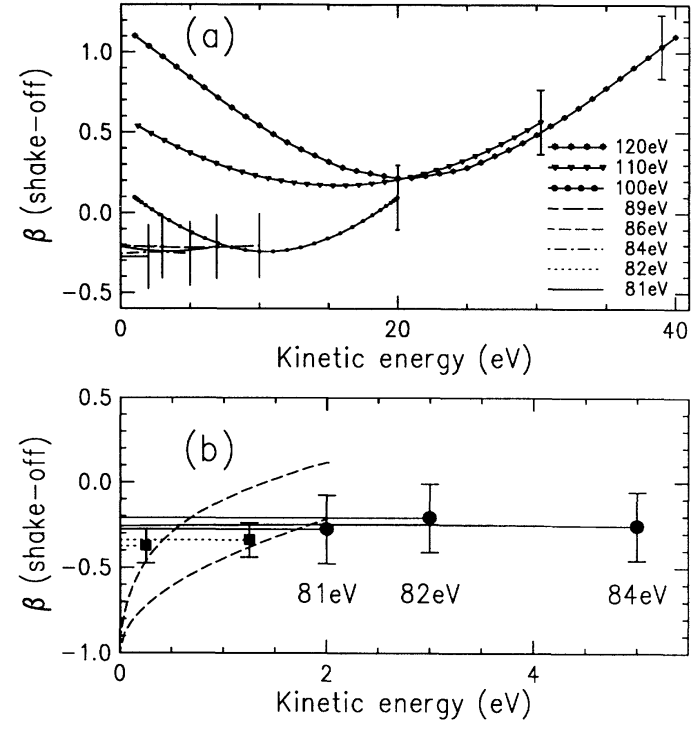

FIG. 5. (a) Angular-distribution curves ( $\beta$ curves) at various photon energies for one shake-off electron with respect to the electric vector of the incident synchrotron light. For clarity, only the error bars at the high-energy end of each curve are depicted. (b) Low-energy part of (a) with shake-off $\beta$ curves at the three lowest photon energies (solid lines with solid circles at the high-energy end). Two representative $\beta$ values chosen from the zero-volt measurements by Hall et al. [13] are depicted by dotted curves assuming flatness of the corresponding angulardistribution $\beta$ curves. For consistency with our data representation the high-energy end of these dotted lines is marked with solid squares. The dashed lines represent two theoretical calculations done by Huetz et al. [14] using different angular correlation factors. Both lines represent $\beta$ values of the high-energy end of $\beta$ curves when the photon energy changes continuously.

range reported in this Letter is an intermediate regime governed by both electron correlations and symmetries.

In conclusion, we have studied the energy and angular distribution of shake-off electrons of helium from 2 to 41 $\mathrm{eV}$ above the double-ionization threshold. In this photon

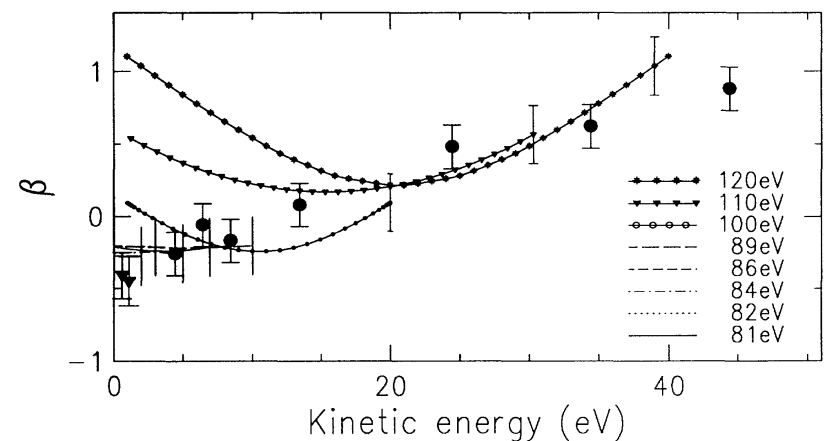

FIG. 6. Shake-off $\beta$ curves at various photon energies as shown in Fig. 5(a) together with $\beta$ values of the satellite $n=4$ (solid circles) on its kinetic energy scale. The triangles represent data from Heimann et al. [27]. 
energy range, shake-off electrons are mainly influenced by electron correlations, while the Wannier range is valid within an energy range of approximately $2 \mathrm{eV}$ above threshold which is in agreement with other publications. We show the first direct experimental evidence that the energy-distribution curves are flat in an extended range above threshold but become $U$-shaped at higher photon energies as expected by theory even without special inclusion of threshold laws. The energy distribution is symmetric with respect to the middle of the curve at the photon energies investigated.

The noncoincident $\beta$ curve is also $U$-shaped and turns to a flat distribution when approaching the doubleionization threshold. The $\beta$ value near threshold is found to be higher than expected by theories based on Wannier's law, a result that may be explained by the competition between electron correlations and symmetry requirements near threshold.

The authors thank Professor Sonntag and his research group for their continuous interest and help at the beam line. We also want to thank Dr. D. Kilcoyne for a critical reading of the manuscript. This work was supported by the Bundesminister für Forschung und Technologie under Contract No. 05414 CAB 7 and by the Deutsche Forschungsgemeinschaft under Contract No. Be860/2-4.

[1] R. P. Madden and K. Codling, Phys. Rev. Lett. 10, 516 (1963).

[2] J. A. R. Samson, Phys. Rev. Lett. 22, 693 (1969).

[3] U. Becker, R. Wehlitz, O. Hemmers, B. Langer, and A. Menzel, Phys. Rev. Lett. 63, 1054 (1989).

[4] J. Mazeau, P. Selles, D. Waymel, and A. Huetz, Phys. Rev. Lett. 67, 820 (1991).

[5] T. A. Carlson, Phys. Rev. 156, 142 (1967).

[6] T. N. Chang and R. T. Poe, Phys. Rev. A 12, 1432 (1975).

[7] G. H. Wannier, Phys. Rev. 90, 817 (1953).
[8] H. Kossmann, V. Schmidt, and T. Andersen, Phys. Rev. Lett. 60, 1266 (1988).

[9] P. Lablanquie, K. Ito, P. Morin, I. Nenner, and J. H. D. Eland, Z. Phys. D 16, 77 (1990).

[10] S. Cvejanović and F. H. Read, J. Phys. B 7, 1841 (1974).

[11] F. Pichou, A. Huetz, G. Joyez, and M. Landau, J. Phys. B 11, 3683 (1978).

[12] G. A. Keenan, I. C. Walker, and D. F. Dance, J. Phys. B 15,2509 (1982).

[13] R. I. Hall, L. Avaldi, G. Dawber, M. Zubek, K. Ellis, and G. C. King, J. Phys. B 24, 115 (1991).

[14] A. Huetz, P. Selles, D. Waymel, and J. Mazeau, J. Phys. B 24, 1917 (1991).

[15] A. Duguet, C. Dupré, and A. Lahmam-Bennani, J. Phys. B 24, 675 (1991).

[16] R. Bruhn, E. Schmidt, H. Schröder, B. Sonntag, A. Thevenon, G. Passereau, and J. Flamand, Nucl. Instrum. Methods 208, 771 (1983).

[17] U. Becker, D. Szostak, H. G. Kerkhoff, M. Kupsch, B. Langer, R. Wehlitz, A. Yagishita, and T. Hayaishi, Phys. Rev. A 39, 3902 (1989).

[18] R. L. Brown, Phys. Rev. A 1, 586 (1970).

[19] T. N. Chang, T. Ishihara, and R. T. Poe, Phys. Rev. Lett. 27, 838 (1971)

[20] S. L. Carter and H. P. Kelly, Phys. Rev. A 16, 1525 (1977).

[21] H. Le Rouzo and C. Dal Cappello, Phys. Rev. A 43, 318 (1991).

[22] V. Schmidt, N. Sandner, H. Kuntzemüller, P. Dhez, F. Wuilleumier, and E. Källne, Phys. Rev. A 13, 1748 (1976).

[23] G. R. Wight and M. J. Van der Wiel, J. Phys. B 9, 1319 (1976).

[24] D. M. P. Holland, K. Codling, J. B. West, and G. V. Marr, J. Phys. B 12, 2465 (1979).

[25] C. N. Yang, Phys. Rev. 74, 764 (1948).

[26] Ch. H. Green, J. Phys. B 20, L357 (1987).

[27] P. A. Heimann, U. Becker, H. G. Kerkhoff, B. Langer, D. Szostak, R. Wehlitz, D. W. Lindle, T. A. Ferrett, and D. A. Shirley, Phys. Rev. A 34, 3782 (1986). 